\title{
Comportamento de estridulação em Heilipus odoratus Vanin \& Gaiger (Coleoptera, Curculionidae, Molytinae)
}

\author{
Adilson Benchaya Nunes ${ }^{1}$, Beatriz Ronchi-Teles ${ }^{2} \&$ Wilson Spironello ${ }^{3}$
}

${ }^{1}$ Instituto de Criminalística do Estado do Amazonas, Avenida Noel Nutels, Cidade Nova, 69090-000 Manaus-AM, Brasil. adilson_benchaya@hotmail.com

${ }^{2}$ Instituto Nacional de Pesquisas da Amazônia, Caixa Postal 478, 69060-020 Manaus-AM. ronchi@inpa.gov.br

${ }^{3}$ wilson@inpa.gov.br

\begin{abstract}
Stridulation behaving in Heilipus odoratus Vanin \& Gaiger (Coleoptera, Curculionidae, Molytinae). Heilipus odoratus Vanin \& Gaiger, 2005 (Coleoptera, Curculionidae, Molytinae) is considered the main plague of rosewood fruit. Being recently described, the biological aspects of this species are not very well known. This study aimed at contributing for the knowledge of this species' behavior and providing a method for sexing adults based on stridulation. Individuals were divided into two groups: stridulating and non-stridulating. After dissection, stridulation was associated with the sex of each individual. Most males $(92.2 \%, N=115)$ emitted sound by stridulation due to the presence of stridulatory organs on the posterior internal surface of their elytra and penultimate tergite, while none of the females $(N=92)$ have such organs. This result is the first report of stridulatory activity in $H$. odoratus.
\end{abstract}

KEYWORDS. Coleoptera; Curculionidae; stridulation.

RESUMO. Comportamento de estridulação em Heilipus odoratus Vanin \& Gaiger (Coleoptera, Curculionidae, Molytinae). A espécie Heilipus odoratus Vanin \& Gaiger, 2005 (Coleoptera, Curculionidae, Molytinae) é considerada o principal inseto-praga de frutos de Pau-rosa (Aniba rosaeodora, Ducke, Lauraceae). Recentemente descrita, pouco se conhece sobre seus aspectos biológicos, tendo este trabalho como objetivo contribuir para o estudo comportamental da espécie, como também fornecer técnicas de sexagem de indivíduos adultos, a partir da estridulação. Para tanto, dividiu-se os indivíduos em dois grupos, os que estridulavam e os que não estridulavam, e estes foram dissecados e a ocorrência de estridulação associada ao sexo de cada indivíduo. Do total de indivíduos observados, a maioria dos machos $(92,2 \%, N=$ 115) emitiu som por estridulação, sendo dotados de órgãos estridulatórios na face interna posterior de seus élitros e no penúltimo tergito, enquanto que a totalidade das fêmeas $(N=92)$ não expressou tal comportamento e são desprovidas de tais aparatos. Este resultado é o primeiro registro da ocorrência de atividade estridulatória em $H$. odoratus.

PALAVRAS-CHAVE. Coleoptera; Curculionidae; estridulação.

Heilipus odoratus Vanin \& Gaiger, 2005 é uma das principais pragas de frutos de Pau-rosa (Aniba rosaeodora Ducke, Lauraceae) (Spironello et al. 2004), espécie aromática da região amazônica produtora de um óleo volátil rico em linalol, substância utilizada na indústria de cosméticos, especialmente como fixadora de perfumes.

As larvas de $H$. odoratus se alimentam das sementes, causando danos irreversíveis, cujo comprometimento dos frutos é bastante significativo, com uma infestação da ordem de $60 \%$ da sua produção em sistemas de plantios (Spironello et al. 2003, 2004).

A espécie apresenta o élitro com duas colorações, sendo duas faixas compostas por escamas de cor amarelada e outra, mais central, de tom marrom avermelhado que é a cor do próprio tegumento, sem concentração de escamas. Heilipus odoratus apresenta dimorfismo sexual pouco evidente, com o comprimento da fêmea variando de 9,3 a 13,5 mm e o macho 10,7 a 12,1 mm; o rostro ligeiramente mais longo e mais delgado na fêmea, com pontuações enfraquecidas e o ventrito $\mathrm{V}$ com o ápice achatado nos machos e convexo nas fêmeas (Vanin \& Gaiger 2005).

A estridulação em Coleoptera ocorre em Carabidae, Scarabaeidae, Tenebrionidae e Curculionidae, sendo verificada também em outras famílias (Chapman 1982), e pode ser definida como o processo pelo qual se dá a produção de som, a partir da fricção de duas partes do corpo do inseto, uma contra a outra (Virant-Doberlet \& Cokl 2004).

De acordo com Lyal \& King (1996), o aparato estridulatório é composto por duas estruturas denominadas pars stridens $\mathrm{e}$ plectrum, que diferem entre as espécies quanto à localização e forma.

Os sons produzidos por insetos são, em geral, específicos de cada espécie (Cokl \& Virant-Doberlet 2003), estando frequentemente relacionados ao comportamento reprodutivo, em muitos casos apenas os machos estridulam (Chapman 1982). Em outros, como nos curculionídeos Erodiscus proximus (Viana 1959) e Arniticus hylobioides (Boheman 1843) observados por Gaiger \& Vanin (2006), a estridulação se dá em ambos os sexos.

Além da função sexual, a estridulação pode exercer também papel de defesa (Virant-Doberlet \& Cokl 2004), na localização de hospedeiros e agregação (Haynes \& Yeargan 1999), marcação de território (Yack et al. 2001), conflito entre membros do mesmo sexo, alarme e sinais pré-copulatórios ou copulatório entre os sexos (Gaiger \& Vanin 2006).

Devido à importância econômica do Pau-rosa e 


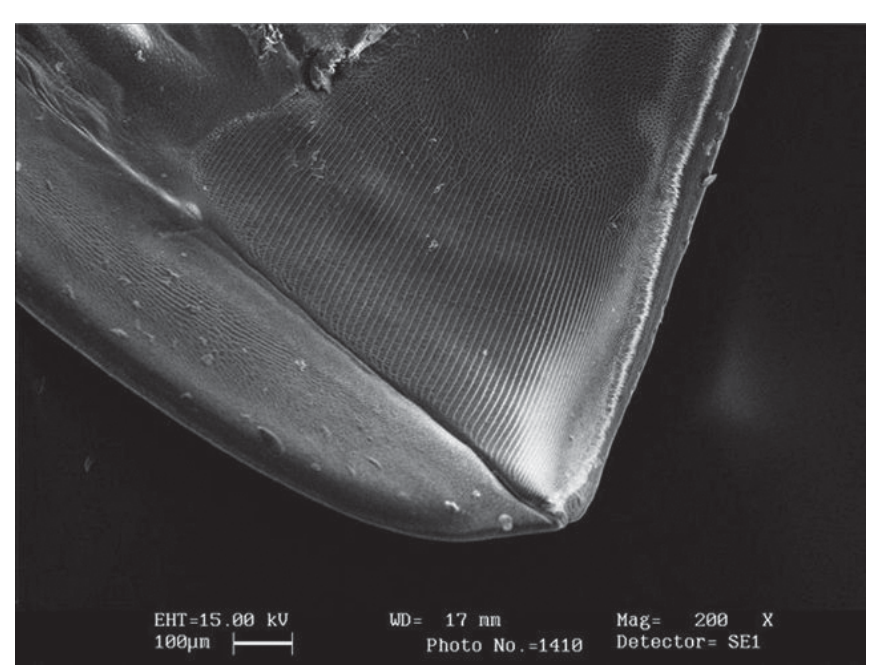

Fig. 1. Pars stridens localizado na face interna do élitro esquerdo de macho de $H$. odoratus.

considerando o desconhecimento da biologia e do comportamento de $H$. odoratus, este estudo tem como objetivo testar se o comportamento estridulatório desta espécie está associado ao sexo dos indivíduos e buscar outras diferenças estruturais entre machos e fêmeas e contribuir para a identificação de sexo de adultos.

A estridulação de $H$. odoratus foi induzida mecanicamente em indivíduos vivos no laboratório a partir de uma leve pressão dorsal ou lateral no abdome, concomitantemente à aproximação de cada indivíduo ao ouvido, uma vez que a emissão sonora é audível sem a necessidade de aparelhos de amplificação.

Para a sexagem dos adultos, 207 indivíduos foram separados em dois grupos: indivíduos que estridulavam e indivíduos que não estridulavam. Os indivíduos de cada grupo, após serem sacrificados em acetato de etila, tiveram seus abdomes retirados e fervidos em ácido lático para posterior dissecação. Após a dissecação, a estridulação foi associada à genitália de cada indivíduo, de acordo com Vanin \& Gaiger (2005), e os aparatos estridulatórios, assim como as diferenças morfológicas, foram registrados em fotografias de microscopia eletrônica de varredura LEO 435 VP ZEISS.

Do total de indivíduos observados, 115 foram machos e 92 fêmeas. A maioria dos machos $(92,2 \%)$ ao serem manipulados produziu som intermitente. Os machos que não estridularam, apesar de possuírem órgãos estridulatórios, apresentavam imperfeições morfológicas dos élitros, estando estes espaçados entre si na porção posterior, o que impedia o contato entre as partes do corpo envolvidas nessa atividade.

A totalidade das fêmeas observadas não expressou tal comportamento e ao contrário dos machos, são desprovidas de órgãos estridulatórios.

Os órgãos estridulatórios de $H$. odoratus são compostos por duas partes: o pars stridens localizado na face interna posterior dos élitros, formando uma série de cristas paralelas (Figuras 1 e 2), prolongando-se quase que perpendicular ao eixo longitudinal do corpo do inseto, e o plectrum ou raspador no dorso do penúltimo tergito que diferentemente das fêmeas

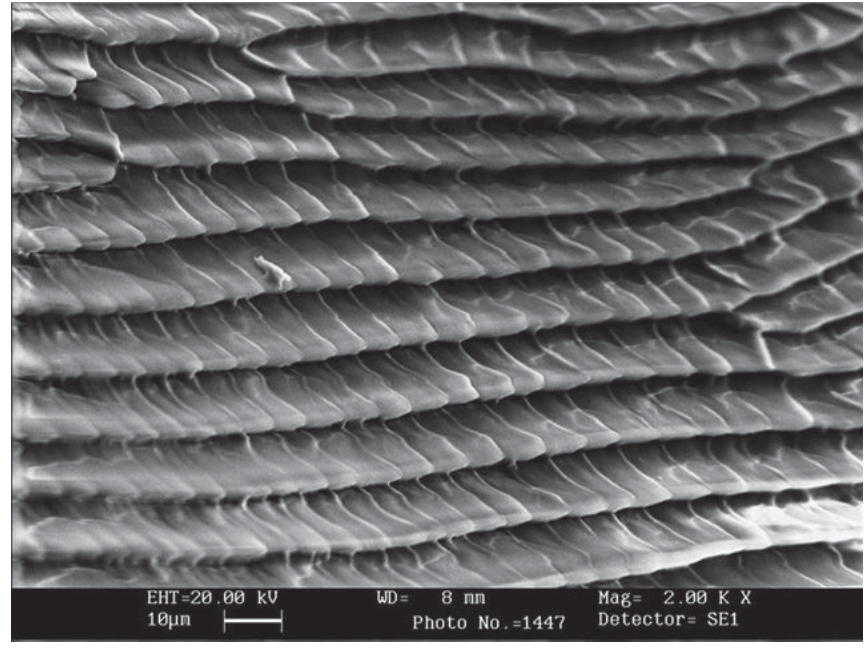

Fig. 2. Visão aproximada do pars stridens de macho de H. odoratus.

(Figura 3), apresenta-se com uma reentrância em sua margem mediana posterior visível a olho desarmado (Figura 4).

A emissão dos sinais acústicos em machos de $H$. odoratus, do tipo élitro tergal, repete-se algumas vezes seguidas, havendo um espaço temporal entre um sinal e outro, realizada a partir da projeção do abdome e posterior retração.

Como apenas machos adultos estridulam, é provável que esta atividade esteja associada ao comportamento reprodutivo, a exemplo do que ocorre com Conotrachelus psidii Marshal, 1922 (Curculionidae), em que os machos estridulam com maior freqüência durante a corte (Silva-Filho et al. 2007).

Mampe \& Neunzig (1966), estudando Conotrachelus nenuphar (Herbst, 1797) (Curculionidae), constataram a fonotaxia intra-específica entre indivíduos presos em armadilhas a outros do sexo oposto, em contraposição de armadilhas que continham indivíduos destituídos dos élitros, onde se localizava o pars stridens.

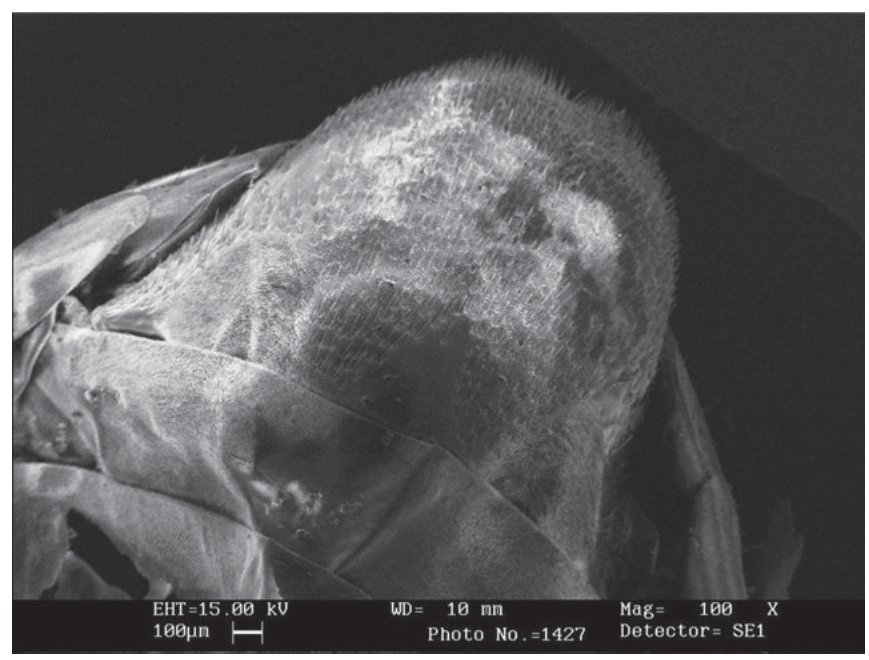

Fig. 3. Penúltimo tergito de fêmeas de $H$. odoratus com a margem posterior reta. 


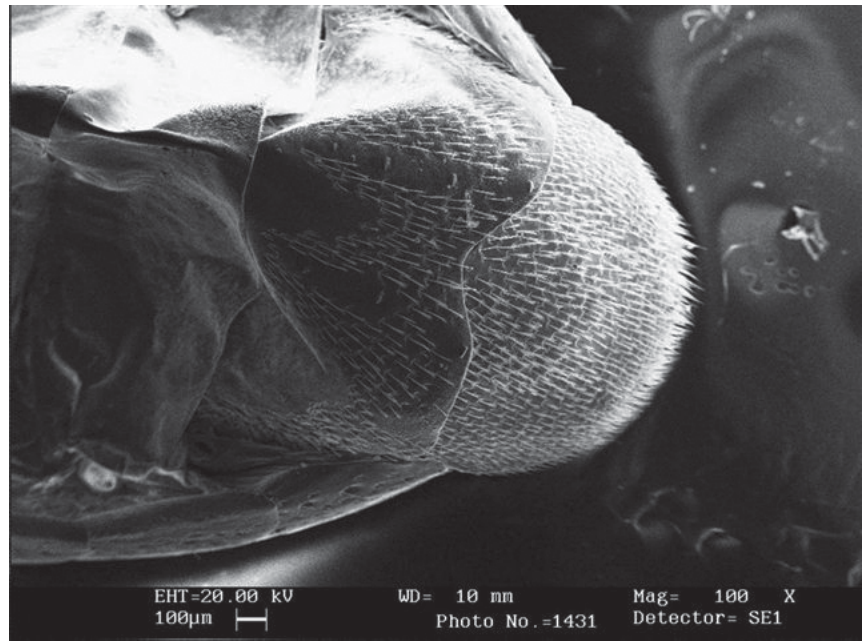

Fig. 4. Plectrum localizado no penúltimo tergito de machos de $H$. odoratus.

De acordo com Schmitt \& Traue (1990) e Wilson et al. (1993) a estridulação, no entanto, é frequentemente relatada como um comportamento de defesa, como em Onyxacalles ringeli Kulbe 1999 (Curculionidae), haja vista que o som só é emitido quando o inseto é tocado.

No caso de H. odoratus, não se pode afirmar o mesmo para a estridulação produzida somente por machos, não obstante ser uma reação observada em laboratório, portanto, induzida pela manipulação mecânica do inseto. O efeito dessa expressão como comportamento de defesa haveria de compreender a espécie como um todo, machos e fêmeas.

Dessa forma, fica reforçada a hipótese do efeito fonotático como expressão de comunicação intra-específica, denotando que a emissão de sons de frequiência $(\mathrm{Hz})$ definida, produzida exclusivamente por machos da espécie, provavelmente seja efeito de comunicação vibracional à curta ou longa distância, possivelmente associado à comunicação química por feromônio. Esse efeito envolve custo energético mínimo e desfavorecimento em sua localização por predadores e parasitóides, composição de estratégias evolutivas estas, inseridas no contexto ecológico do sucesso do estabelecimento, isolamento e perpetuação da espécie.

Finalizando, o comportamento estridulatório de $H$. odoratus é uma importante ferramenta para a sexagem de indivíduos adultos vivos (audição do som emitido) ou mortos (pelo dimorfismo sexual do aparato estridulatório), assim como fornecer indícios de qual sexo está envolvido na atração do sexo oposto e/ou co-específicos. Tal informação poderá servir de alicerce para estudos comportamentais relacionados à reprodução e posterior desenvolvimento de métodos de controle e monitoramento da espécie com o uso de armadilhas sonoras.
Agradecimentos. Sérgio Antonio Vanin (Instituto de Biociências da Universidade de São Paulo) e Fábio Gaiger Silveira (Ministério da Saúde), pela identificação da espécie; José Wilson Meirelles pelas fotografias de microscopia eletrônica de varredura; Instituto Nacional de Pesquisas da Amazônia pelo apoio logístico, ao $\mathrm{CNPq}$ pela bolsa concedida ao primeiro autor, e a FAPEAM pelo apoio financeiro.

\section{REFERÊNCIAS}

Chapman, R. F. 1982. The insects: structure and function, Cambridge Harvard University Press iii+919 p.

Cokl, A. \& M. Virant-Doberlet. 2003. Communication with substrateborne signals in small plant-dwelling insects. Annual Review of Entomology 48: 29-50.

Gaiger, F. \& S. A. Vanin. 2006. The elytro-femoral stridulatory apparatus in Curculionidae (Coleoptera), with notes on the acoustic behavior of Arniticus hylobioides (Boheman 1843) and Erodiscus proximus (Viana 1959), and thanatosis display in the latter species. Annales de la Société Entomologique de France 42: 165-170.

Haynes, K. F. \& K. V. Yeargan. 1999. Exploitation of intraspecific communication systems: elicit signalers and receivers. Annals of the Entomological Society of America 92: 960-970.

Lyal, C. H. C. \& T. King. 1996. Elytro-tergal stridulation in weevils (Insecta: Coleoptera: Curculionoidea). Journal of Natural History 30: 703-773.

Mampe, C. D. \& H. H. Neunzig. 1966. Function of the stridulating organs of Conotrachelus nenuphar. Annuals of the Entomological Society of America 59: 614-615.

Schmitt, M. \& D. Traue. 1990. Morphological and bioacoustic aspects of stridulation in Criocerinae (Coleoptera: Chrysomelidae) Zoologischer Anzeiger 225: 225-240.

Silva-Filho, G.; O. E. Bailez \& A. M. Viana-Bailez. 2007. Dimorfismo sexual do Gorgulho-da-Goiaba Conotrachelus psidii Marshall (Coleoptera: Curculionidae). Neotropical Entomology 36: 520 524.

Spironello, W. R.; P. T. B. Sampaio; G. Vieira \& A. Barbosa. 2003. Ecologia reprodutiva do Pau-rosa (Aniba rosaeodora Ducke) em mata de terra firme na Amazônia Central. p. 69-87. In: N. Higuchi; J. dos Santos; P. T. B. Sampaio; R. A. Marrenco; J. Ferraz; P. C. de Sales; M. Saito; S. Matsumoto (Eds.) Projeto Jacaranda - Fase II: Pesquisas Florestais na Amazônia Central. Manaus, INPA, $252 \mathrm{p}$.

Spironello, W. R.; P. T. B. Sampaio \& B. Ronchi-Teles. 2004. Produção e predação de frutos em Aniba rosaeodora Ducke var. Amazonica Ducke (Lauraceae) em sistema de plantio sob floresta de terra firme na Amazônia Central. Acta Botanica Brasilica 18: 801807.

Vanin, S. A. \& F. Gaiger. 2005. A new spermophagous species of Heilipus Germar from the Amazonian Region (Coleoptera, Curculionidae, Molytinae). Revista Brasileira de Entomologia 49: 240-244.

Virant-Doberlet, M. \& A. Cokl. 2004. Vibrational communication in insects. Neotropical Entomology 33: 121-134.

Wilson, L. M.; C. S. Henry; J. B. Johnson \& J. P. Mccaffrey. 1993 Sound production in Phrydiuchus tau (Coleoptera: Curculionidae) Annals of the Entomological Society of America 86: 621630.

Yack, J. E.; M. L. Smith \& P. J. Weatherhead. 2001. Caterpillar talk: acoustically mediated territoriality in larval Lepidoptera. Proceeding of Natural Academy of Sciences of USA 98 : 11371-11375. 